\title{
LIII. Some new experiments with the flame tube
}

\section{U. Behn}

To cite this article: U. Behn (1907) LIII. Some new experiments with the flame tube, Philosophical Magazine Series 6, 13:77, 627-630, DOI: 10.1080/14786440709463639

To link to this article: http://dx.doi.org/10.1080/14786440709463639

曲 Published online: 16 Apr 2009.

Submit your article to this journal 정

Џll Article views: 5

Q View related articles $₫$ 


\section{$\left[\begin{array}{ll}627 & 0\end{array}\right.$ \\ LIII. Some new Experiments with the Flame Tube. By U. BEHN *.}

7 THE first experiments with the flame-tube were made to show the change of air-pressure with height and for some meteorological demonstrations $\dagger$. Most elegantly it has been used by Mr. Rubens $\ddagger$, and by Messrs. Rubens and Krigar-Menzel $\S$ in acoustics.

In order to obtain the maximum sensitiveness of the flametube, which was not fally appreciated even by Mr. Rubens $\ddagger \S$, the friction in the tube must be as small as possible. Hence it has been pointed out as of first importance, that the tube and the outlet holes should be wide $\left(e_{0} g\right.$. , tube not less than $5 \mathrm{~cm}$. diameter and the holes 5 to $1 \mathrm{~cm}$. diam.). If these conditions are not fulfilled, as recently occurred II, the sensitiveness of the tube will of course be spoiled.

The simple method of using the flame-tube to show the change of the pressure of the atmosphere with the height may be again described here. A metal tube of suitable length is closed at the ends. At $\mathrm{C}$ (fig. 1) is an opening,

Fig. 1.

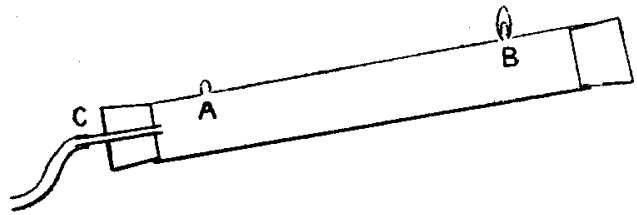

connected by a flexible tube to the gas mains. A and B are two holes of equal diameter (instead of having only two holes, there may be a number spaced equidistantly). Gas is allowed to pass into the tube (turned over to prevent

* Communicated by the Author.

† U. Behn, Z. S. f. d. phys. u. chem. Unterricht, xvi. p. 129 (1903).

t. Ber. d. Deutsch. Physic. Ges. p. 351 (1904).

\$ Ann. d. Physik, xvïi. p. 149. (1905).

Regarding the theory compare Lord Rayleigh's paper "On the Circulation of Air observed in Kundt's Tubes and on some allied Acoustical Problems." Phil. Trnns. clxxv. p. 1 (1883).

II P. Steindel, Z. S. f. d. phys. u. chem. Unterr. 19.

In acoustics the best results are obtained with notes of high pitch. Mr. Rubens chose, sacriticing part of the sensitiveness, the holes relatively small to extend the application to notes of some hundreds oscillation. 
escape from the holes) and is then lit at the openings. The flow is adjusted so that there is a small luminous tip to the flames, the tube being meanwhile supported in a horizontal position.

If the tube is now slightly tilted, so that one end, say A, is higher than the other, it will be found that the flame at the higher end of the tube becomes comparatively large and that at the lower end comparatively small. The same effect is obtained, whichever end of the tube is raised, so it cannot be caused by inequality in the size of the holes, or by friction in the tube. (At A the gas-pressures inside and outside the tube are sensibly equal, as is shown by the small size of the flame. Above A the pressure decreases more quickly in the dense air than in the light coal-gas. Hence at $B$ the pressure inside the tube exceeds the pressure outside, and so the flame is large.) If one end lies only three millimetres higher than the other, a difference in the size of the flames will be produced, which, if the air is undisturbed, may be plainly seen from all over a large lecture-hall $(15-20 \mathrm{~m}$.). In this way a difference in pressure of about ( $\cdot 0012-0006) \cdot 03$ gram weight per sq. cm., or $\cdot 000,000,2$ atmosphere ${ }^{*}$ is made visible.

A new field of application for the flame-tube lies in its use for indicating small motions, or rather small accelerations. A slight movement of the tube causes changes of pressure in gas, due to the latter's inertia, and these changes of pressure in the tube are made visible by the difference in the sizes of the flames. For example, the beats of a seconds pendulum, though they be very small, may be made visible to the naked eye. For this purpose the flame-tube is suspended like a pendulum. The tube for these experiments need only be about $.5 \mathrm{~m}$. long $\dagger$ and one flame-hole at either end is sufficient. On account of the experiments described further on, the tube should be as light as possible. For the experiment in question, one ought to increase its weight by putting a $n$-shaped piece of lead sheeting on it (fig. 2). Oscillations of one $\mathrm{cm}$. amplitude will suffice to make the jumping of the flames visible from all over a lecture theatre.

* Approximately; the difference of temperature of coal-gas and surrounding air being not very large.

$\dagger$ In experiments, for which, on the contrary, a very long flame-tube is required, it is sometimes advisable to build the tube up by a number of pieces put loosely together. In this way one may avoid the bending of the tube, which of course is stronger heated by the flames from the upper side. 
If one wants to show the kind of motion during the oscillations of a pendulum, one preferably suspends the

Fig. 2.

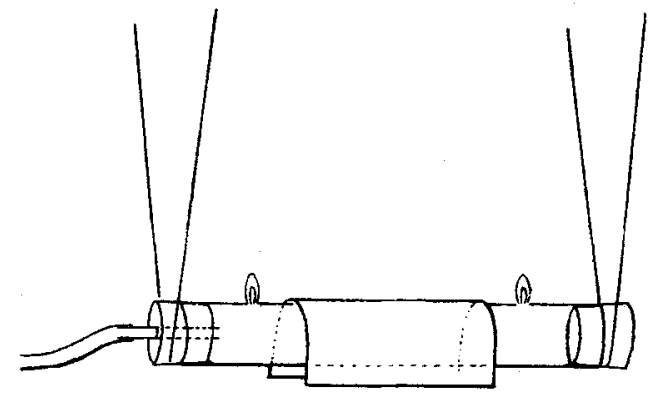

flame-tube by longer threads, and uses larger amplitudes. Then it is easily seen, that the changes in the sizes of the flames do not take place, when the pendulum is at the point of turning back, but when it is near the position of rest, where the acceleration changes sign.

If another pendulum is hang on to the swinging flametube (or vice versa) and the length of both pendulums suitably adjusted, the changes of the flames show how the simple swinging mation is altered by the addition of the octave, twellth, etc.

As one can see from the foregoing experiment the flametube pendulum might prove of use in detecting recoils. Thus a disk (of cardboard) whose centre of gravity may be displaced from the geometric centre by fastening a small weight to it near the circumference, is fastened to the flametube. Rotating the disk (taking care not to swing the flame-tube by the impulse) the flames will remain undisturbed until the weight is brought into play, when the oscillatory reaction becomes noticeable even though the weight may amount to less than one-hundredth of the total weight.

Similarly the reactionary motion caused by a very small pendulum may be shown. A pendulum consisting of a shot suspended by a thread will be sufficient.

It can also be shown by means of the flame-tube pendulum, that a system originally at rest cannot alter the position of its centre of gravity, unless acted on by an external force (e. g., case of the recoil of a gun). For instance, a steel band, the shape of a large tuning-fork, is fastened to the 
flame-tube pendulum as shown in fig. 3 and bent by tying the two ends at $(a)$ together. The thread joining the two

Fig. 3.

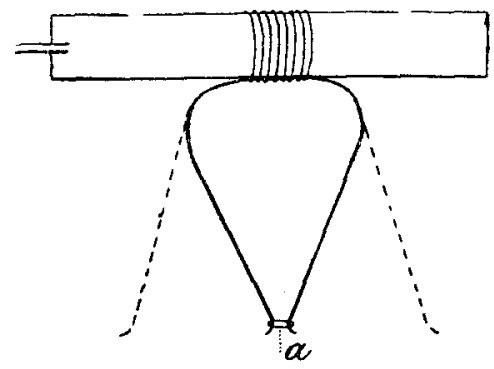

ends at $(a)$ is burnt, and the spring thus set in motion. If the band is geometrically and physically perfectly symmetrical, the flames remain at rest *.

Another case, where inertia plays a rôle, we have in the so-called centrifugal force. If the flame-tube lying on the table is turned about one end as centre, the flame at the other end becomes large through the centrifugal force causing an increase of the internal pressure at that end of the tube. This effect is shown even if the motion is very slow.

The experiments described above do not of course exhaust the uses of the flame-tube, but merely serve as examples.

Physical Laboratories,

Victoria University, Manchester.

LIV. The Decomposition of Water-Vapour in contact with Hot Platinum Wire. By Alfred Holt, Junr. $\dagger$

D ATHER more than a year ago the author made several R attempts to estimate the extent of the dissociation of water-vapour at high temperatures by heating a platinum wire (by means of an electric current) in contact with it at low pressures. The results were consistent among themselves, but at high temperatures were decidedly lower than those previously obtained by Nernst and Wartenberg (Göttinger Nachr. 1905, p. 35). The work was not published, owing to the circumstance that some doubt arose as to whether the numbers were a true measure of the dissociation of the vapour at the temperature of the wire.

* A cork pushed in between the prongs at (a) serves well enough for holding the axle for the disk used in the last experiment.

+ Communicated by the Author. 